natorgüte $Q$ eintritt, bei zu kleinem Querschnitt aber das Füllvolumen zu klein wird. Bei Wasserproben z. B. hat FeHER ${ }^{15}$ gezeigt, daß man optimale Empfindlichkeit erreicht, wenn die Wassermenge die Resonatorgüte $Q$ auf $2 / 3$ verringert. Dies wurde bei Ammoniak bei ca. $1,7 \mathrm{~mm}$ Innendurchmesser erreicht.

5. Die Verwendung von Quarz- und Glasröhrchen bei ESR-Messungen

Quarz hat im allgemeinen geringere dielektrische Verluste als Glas. Außerdem besteht bei Glas die Ge- fahr, daß es paramagnetische Beimischungen enthält. Es wurde aber festgestellt, daß zwischen Probenröhrchen aus Quarz und aus Jenaer Glas für ESR-Messungen praktisch kaum ein Unterschied besteht. Aus diesem Grunde und wegen der beträchtlichen Erleichterung der glasbläserischen Arbeiten wurde hier ausschließlich Jenaer Glas verwendet.

Für die Förderung dieser Arbeit danken die Verfasser dem Direktor des Physikalisch-Chemischen Instituts, Herrn Prof. Dr. G. Scheibe sowie dem B u n d e s ministerium für wissenschaftliche For. $\mathrm{schung}$.

15 G. FEHER, Bell. System techn. J. 36 [1957].

\title{
Innermolekulare Wasserstoffbrücken und Tieffarbigkeit bei den Fluorenon-1-carbonsäuren
}

\author{
Von Günther Demmering * und Friedrich Dörr \\ Aus dem Institut für physikalische Chemie der Technischen Hochschule München \\ (Z. Naturforschg. 19 b, 365—375 [1964] ; eingegangen am 18. Dezember 1963)
}

\begin{abstract}
Es wurden die UV-Absorptionsspektren der Lösungen verschiedener Fluorenon-carbonsäuren aufgenommen. In neutraler Lösung ist die sogenannte $n \rightarrow \pi^{\mathrm{x}}$-Bande der Ketogruppe die längstwellige Elektronenbande. Das Spektrum der Fluorenon-1-carbonsäure wird mit zunehmender Acidität des Lösungsmittels nach langen Wellen verschoben; die für das Auge dabei wahrnehmbare Verfärbung nach Rot rührt von einer Absorptionsbande her, die nach Intensität und Form die langwellig verschobene Ketobande zu sein scheint. Ähnlich wie das Spektrum in saurer Lösung verläuft das Remissionsspektrum der mit $\mathrm{NaCl}, \mathrm{KCl}, \mathrm{Al}_{2} \mathrm{O}_{3}$ oder $\mathrm{MgO}$ verriebenen festen Substanz.

Im IR-Spektrum der kristallinen Fluorenon-1-carbonsäure und bei Verwendung von $\mathrm{CHCl}_{3}$ als Lösungsmittel wurden durch Vergleich mit dem Silbersalz und durch Deuterierung 6 OH-Valenzbanden identifiziert, die durch Wasserstoffbrückenbindung langwellig verschoben sind. Die scharfen OH-Banden verschwinden in Dimethylsulfoxyd als Lösungsmittel.

Es wird geschlossen, daß in der kristallinen Fluorenon-l-carbonsäure eine innere Wasserstoffbrücke zwischen Carboxyl-OH und Keto-O mit zwei nahezu gleich tiefen Potentialmulden vorliegt. Die tiefe Farbe scheint mit einer oxoniumähnlichen Struktur der Ketogruppe zusammenzuhängen.
\end{abstract}

Die Kristalle der Fluorenon-1-carbonsäure (II) sind zinnoberrot, während die der Fluorenon-3-(III) und der Fluorenon-4-carbonsäure (IV) gelb sind, wie das Fluorenon (I) selbst.
Die Ester und Salze der Fluorenon-1-carbonsäure sind als Kristalle ebenfalls gelb.

Diese „optische Anomalie“ wurde von $\mathrm{H}_{\text {AnTzSCH }}{ }^{1}$ mit einer Wechselwirkung der benachbarten Carbo-

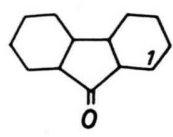

$I$

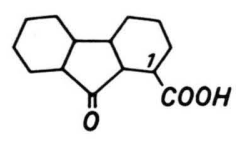

II

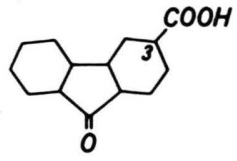

III

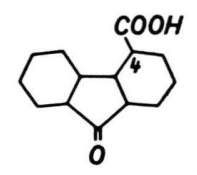

IV

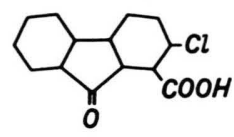

$\boldsymbol{\nabla}$

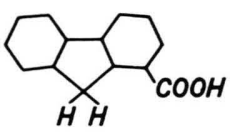

प

* Auszug aus der Dissertation des Diplomchemikers Günther Demmering, TH München 1963.

1 A. Hantzsch, Ber. dtsch. chem. Ges. 49, 226 [1916]. 
nyl- und Carboxylgruppe in der Fluorenon-1-carbonsäure (II) erklärt, durch die sich ein ,inneres Oxoniumsalz" ausbilden soll. Dieser Schluß wird nahegelegt durch das Verhalten der 1-Säure (II) in Lösung: während die alkoholische Lösung gelb ist, ist die Lösung in Trifluoressigsäure, mit der sich sehr wahrscheinlich ein ,äußeres“ Oxoniumsalz bildet, zinnoberrot. Bei einer neueren Untersuchung fanden Tröster $^{2}$ und Sieglitz ${ }^{3}$ im IR-Spektrum der gelben Verbindungen III und IV je eine OH-Bande, bei der roten Verbindung (II) zwei OH-Banden, die TröSTER $^{2}$ einer zwischenmolekularen bzw. einer inneren H-Brücke zuordnet. Daß die 2-Chlor-Fluorenon-1carbonsäure (V) wieder gelb ist, erklärt TRösTeR ${ }^{2}$ mit einer Störung der innermolekularen H-Brücke durch das benachbarte Chloratom.

Im folgenden wird zunächst untersucht, ob die tiefe Farbe von II von einer $n \rightarrow \pi^{x}$. oder einer $\pi \rightarrow \pi^{x}$. Elektronenbande herrührt. Dann wird versucht, aus den mit höherer spektraler Auflösung als bei TRöSTER $^{2}$ aufgenommenen OH- und CO-Valenzschwingungsbanden einige Aussagen über die Struktur der inneren Wasserstoffbrücke zu gewinnen.

\section{UV-Absorptionsspektren in verschiedenen Lösungsmitteln}

Die im festen Zustand zinnoberrote Fluorenon-1carbonsäure (II) löst sich in Tetrachlorkohlenstoff, Chloroform, Alkohol und Dimethylsulfoxyd mit gelber Farbe. Dabei beobachtet man mit zunehmender Polarität des Lösungsmittels eine wachsende Verschiebung der schwachen langwelligen Absorptionsbande, die die Farbe verursacht, nach kurzen Wellen: sie liegt in $\mathrm{CCl}_{4}$ bei 24500, in $\mathrm{CHCl}_{3}$ bei 25 000, in Äthanol bei 26500 und in Dimethylsulfoxyd bei $27000 \mathrm{~cm}^{-1}$.

Das Spektrum der 1-Säure (II) gleicht weitgehend dem der isomeren 4-Säure (IV) und damit auch dem des Fluorenons selbst, dagegen absorbiert (V) bei etwas größeren Wellenlängen als (II). In alkoholischer Lösung liegt das Maximum der „Farbbande" bei $25500 \mathrm{~cm}^{-1}$ (vgl. Abb. 1).

Mit zunehmender Acidität des Lösungsmittels wird die Farbbande der Fluorenoncarbonsäuren und die des Fluorenons selbst nach langen Wellen verscho.

2 H. TRöster, Diss., TH München 1961.

3 A. Sieglitz u. H. Tröster, Chem. Ber. 96, 2577 [1963]. ben. So ist die Lösung von II in Trifluoressigsäure zinnoberrot $\left(23800 \mathrm{~cm}^{-1}\right)$, in $\mathrm{H}_{2} \mathrm{SO}_{4} \quad(20500$ $\mathrm{cm}^{-1}$ ) tiefrot (vgl. Abb. 2).

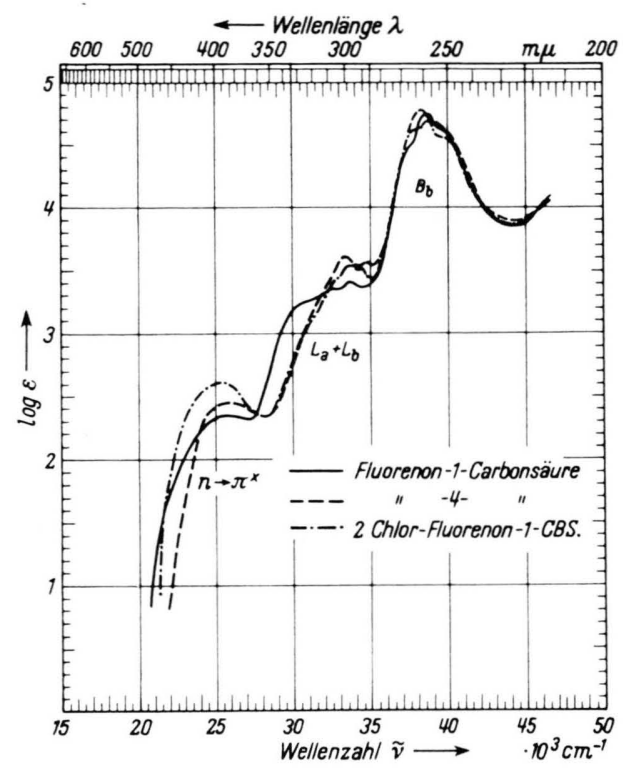

Abb. 1. Absorptionsspektren der Fluorenon-1-carbonsäure, der 2-Chlorfluorenon-1-carbonsäure und der Fluorenon-4-carbonsäure; $c=10^{-4}-m$. in Äthanol.

Die entsprechende Fluorenonbande erscheint von $26500 \mathrm{~cm}^{-1}$ in Äthanol auf $25000 \mathrm{~cm}^{-1}$ in Trifluoressigsäure und $18500 \mathrm{~cm}^{-1}$ in konz. $\mathrm{H}_{2} \mathrm{SO}_{4}$ bathochrom verschoben (vgl. Abb. 3) .

\section{UV-Remissionsspektren}

Um Aufschluß über die Lage der Farbbande in der roten kristallinen Fluorenon-1-carbonsäure zu erhalten, wurden nach der Methode von Ковтüм ${ }^{4}$ die UV-Remissionsspektren in einem geeigneten Verdünnungsmittel aufgenommen. Abb. 4 zeigt u. a. das Spektrum von I (5) und II (4) in standardisiertem (noch $\mathrm{H}_{2} \mathrm{O}$ enthaltendem) $\mathrm{Al}_{2} \mathrm{O}_{3}$. Das Feststoffspektrum zeigt ähnlichen Verlauf wie die entsprechenden Lösungsmittelspektren (Kurven 4 und 5); die Carbonylbande liegt jeweils bei $25000 \mathrm{~cm}^{-1}$. Bei Verwendung von $\mathrm{Al}_{2} \mathrm{O}_{3}$, das bei $900^{\circ}$ getrocknet wurde, oder von $\mathrm{MgO}$ erhält man ein stark verflachtes Spektrum der Säure. Die langwellige Absorption beginnt

\footnotetext{
${ }^{4}$ G. Ковтём, Z. physik. Chem. N.F. 18, 110, 230 [1958]; Angew. Chem. 70,651 [1958].
} 

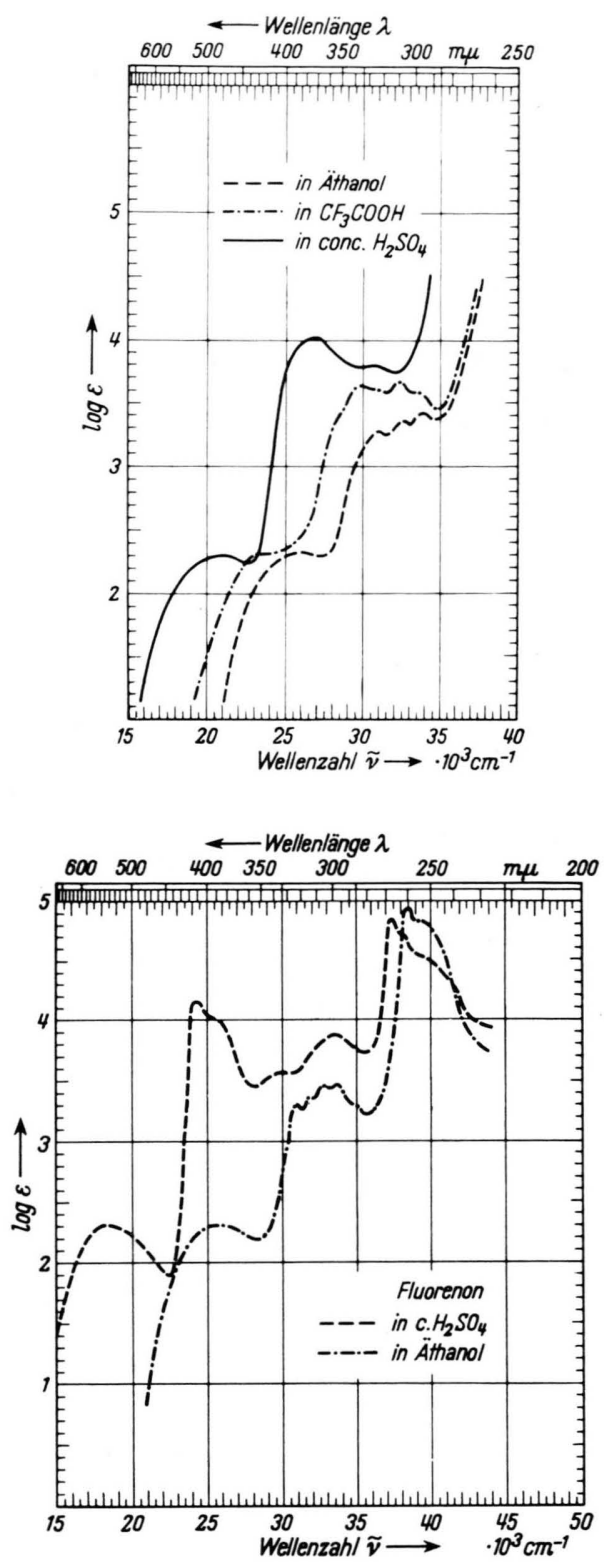

Abb. 2 u. 3. Langwellige Verschiebung der Farbbande der Fluorenon-1-carbonsäure und des Fluorenons durch Säure; $c=10^{-4}-m$. in konz. $\mathrm{H}_{2} \mathrm{SO}_{4}$ bzw. $\mathrm{CF}_{3} \mathrm{COOH}$.

bereits bei $17000 \mathrm{~cm}^{-1}$, das Maximum liegt bei $25000 \mathrm{~cm}^{-1}$ (Kurve 1). Die Spektren des Fluorenons in standard. $\mathrm{Al}_{2} \mathrm{O}_{3}$ und in getrocknetem $\mathrm{Al}_{2} \mathrm{O}_{3}$ unterscheiden sich dagegen praktisch nicht. Das Feststoffspektrum der Fluorenon-1-carbonsäure erscheint also bei Verwendung von getrocknetem $\mathrm{Al}_{2} \mathrm{O}_{3}$ und von $\mathrm{MgO}$ gegenüber den Lösungsspektren stark verändert. Arbeitet man dagegen mit sorgfäl-

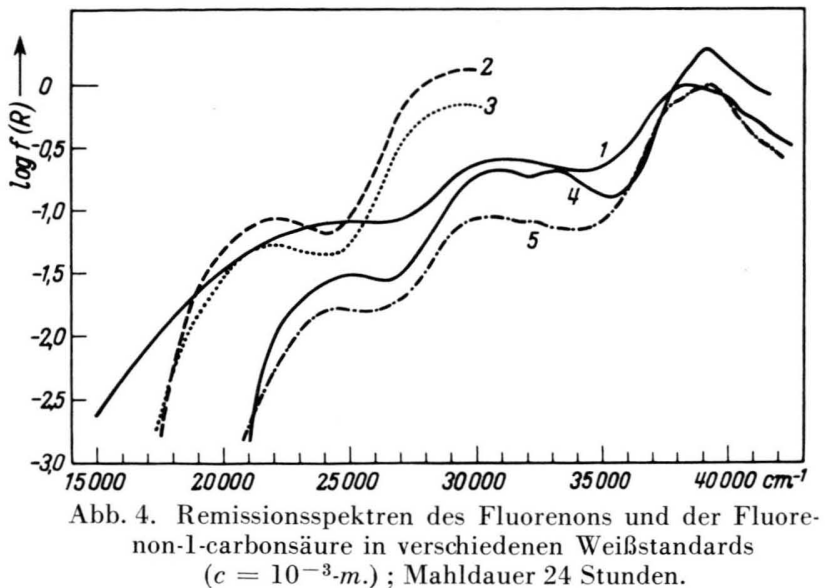

tig getrockneten Alkalihalogeniden als Verdünnungsmittel für die Säure, so findet man die „Farbbande“ verhältnismäßig scharf und gegen die entsprechende Bande des Fluorenons um $3000 \mathrm{~cm}^{-1}$ langwellig verschoben bei $22000 \mathrm{~cm}^{-1}$, wie dies auch der roten Farbe der Kristalle entspricht. Alkalihalogenide können keine entsprechenden Bindungen eingehen, wie sie zwischen der Säure II und dem basischen $\mathrm{Al}_{2} \mathrm{O}_{3}$ möglich sind $(2$ : in $\mathrm{KCl}, 3$ : in $\mathrm{NaBr}$ ).

\section{Diskussion der Elektronenspektren}

\subsection{Zuordnung der Absorptionsbanden}

Die längstwellige Bande des Fluorenons um $25000 \mathrm{~cm}^{-1} \quad(\log \varepsilon<3)$ wird von der Anregung eines der einsamen (n-) Elektronen am Ketosauerstoff in eine leere $\pi$-Bahn des ganzen Molekelgerüsts verursacht ( $\mathrm{n}-\pi$-Bande, Ketobande, Carbonylbande). Die im UV liegenden intensiveren Banden $(\log \varepsilon>3)$ rühren von Übergängen innerhalb der $\pi$-Elektronen her $(\pi-\pi$-Banden $)$. Die Bezeichnung $\mathrm{L}_{\mathrm{a}}, \mathrm{L}_{\mathrm{b}}, \mathrm{B}_{\mathrm{b}}$ deutet auf die Analogie zu entsprechenden Aromatenbanden hin ${ }^{5}$.

Die Anregung in der $n-\pi^{x}$-Bande führt zu einem diradikalähnlichen Zustand, bei dem ein Elektron am Sauerstoff lokalisiert bleibt, während das andere über das Molekelgerüst wandern kann. Die Lage der Carbonylbande ist von der Ladungsdichte am Sauerstoff und in der CO-Bindung bestimmt, die wiederum durch mesomere und induktive Effekte mit den Substituenten bedingt ist. Im Benzophenon ist die Wechselwirkung zwischen den Ringen behindert, weil

\footnotetext{
5 F. Dörr, Habil.-Schrift, TH München 1958.
} 
sie aus sterischen Gründnen nicht in einer Ebene liegen. Im Fluorenon liegt dagegen ein zentraler Fünfring mit entsprechend gespanntem Winkel vor, das Molekül ist eben, die $n \rightarrow \pi^{\mathrm{x}}$-Absorptionsbande des Fluorenons liegt um 3000 Wellenzahlen längerwellig als beim Benzophenon.

Der übrige Teil des Fluorenonspektrums liegt nach Untersuchungen von DörR ${ }^{5}$ zwischen dem des Naphthalins und dem des Phenanthrens. Die $\mathrm{B}_{\mathrm{b}}$-Bande liegt beim Fluorenon an der gleichen Stelle wie im Anthracen und Phenanthren; die bei $30000 \mathrm{~cm}^{-1}$ beginnenden stark strukturierten Banden entsprechen einer Überlagerung der $\mathrm{L}_{\mathrm{a}}$ - und der $\mathrm{L}_{\mathrm{b}}$-Banden des Naphthalins.

Die Spektren der Fluorenoncarbonsäuren haben im Sichtbaren und im UV den gleichen Aufbau und eine sehr ähnliche Lage wie das des Grundkörpers Fluorenon.

\subsection{Lösungsmitteleffekte}

Die für die Fluorenonderivate gefundene „negative Solvatochromie" in polaren Lösungsmitteln (Abschn. 2) ist ganz allgemein für $\mathrm{n} \rightarrow \pi^{\mathrm{x}}$-Banden zu erwarten. Durch polare Lösungsmittel werden die freien Elektronenpaare am Sauerstoff beansprucht und ihre Anregungsenergie vergrößert, da der Grundzustand für $n \rightarrow \pi^{\mathrm{x}}$-Übergänge durch polare Lösungsmittel stärker stabilisiert wird als die Anregungszustände. Aus der Beobachtung, daß das Feststoffspektrum der Fluorenon-1-carbonsäure eine langwellige Bande zeigt (statt 24500 in $\mathrm{CCl}_{4}$ $22000 \mathrm{~cm}^{-1}$ in $\mathrm{KCl}$ ), die der orangeroten Farbe der Säure entspricht, folgt, daß durch die Kristallisation der angeregte Zustand energetisch mehr stabilisiert wird als der Grundzustand. Während in alkoholischer Lösung bei Fluorenon (I) und den isomeren Säuren II, III, IV die Farbbande übereinstimmend bei $26500 \mathrm{~cm}^{-1}$ liegt, erscheint sie in den Feststoffspektren bei verschiedenen Wellenzahlen (I und IV : $25000 \mathrm{~cm}^{-1}$, für II : $22000 \mathrm{~cm}^{-1}$ ). Bei der Fluorenon-1-carbonsäure sind zwei Konformationsisomere a und b mit verschiedenem Energieinhalt möglich.

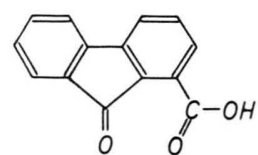

a

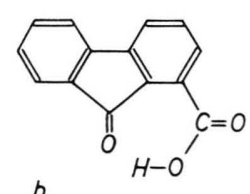

b
Die tiefe Farbe von II hängt offenbar mit der Möglichkeit zusammen, über eine intramolekulare Wasserstoffbrückenbindung einen sterisch und energetisch begünstigten 7-Ring auszubilden. Es ist anzunehmen, daß im Kristall die Form b vorliegt, die durch Ringbildung stabilisiert und daher energieärmer ist. Beim Erwärmen von II beobachtet man eine deutliche Aufhellung. Die Schmelze ist gelb - beim Abkühlen entstehen wieder die orangeroten Nadeln. Trotzdem verschwinden im IR-Spektrum auch in der Schmelze die Wasserstoffbrücken-Schwingungen nicht (vgl. Abschn. 4.2.3). Beim Lösen der Verbindung kommt es zur Ausbildung intermolekularer H-Brücken mit dem Solventen, was mit einer Aufhebung des inneren Chelatringes einhergeht. Die Farbaufhellung in Alkohol und Dimethylsulfoxyd hängt vermutlich damit zusammen; aber auch in $\mathrm{CHCl}_{3}$ und in dem völlig unpolaren $\mathrm{CCl}_{4}$ beobachtet man eine zwar geringere, aber immer noch deutliche hypsochrome Verschiebung der langwelligen Bande gegenüber der kristallinen Substanz.

\subsection{Einfluß von Säuren auf die Spektren der Fluorenon-Derivate}

Im allgemeinen gilt, daß sich die Säureprotonen an die Stellen hoher Elektronendichte - das sind bei den zu untersuchenden Substanzen die einsamen Elektronenpaare am Sauerstoff - anlagern (exakter müßte man die Änderung der freien Energie bei der Protonenanlagerung untersuchen, in die auch die Entropie und die Solvationsenergien eingehen). Da die Anregung einer Carbonylgruppe in der Anregung eben eines solchen n-Elektrons beruht, werden durch Säuren die $n \rightarrow \pi^{x}$-Banden im allgemeinen nach kurzen Wellen verschoben. Dies gilt als ein wesentliches Merkmal von $n \rightarrow \pi^{\mathrm{x}}$-Übergängen. Die Verschiebung der relativ schwachen Carbonylbande nach kurzen Wellen und eine gleichzeitige starke bathochrome Verschiebung der intensiven $\pi \rightarrow \pi^{\mathrm{x}}$. Bande führt bei manchen Ketonen schließlich zu einer Überlagerung der beiden Banden in stark polaren Lösungsmitteln. Die hier untersuchten Substanzen scheinen von dieser Regel abzuweichen. Fluorenon und seine Derivate zeigen in Säuren eine mit der Acidität des Lösungsmittels zunehmende langwellige Verschiebung der "Farbbande“ in den sichtbaren Bereich (vgl. Abb. 3), wodurch die sauren Lösungen farbiger erscheinen. Die Farbintensi- 
vierung ist dabei nicht auf die Fluorenon-1-carbonsäure (II) beschränkt, steht also nicht mit der Möglichkeit der Bildung eines inneren 7-Ringes in $\mathrm{Zu}$ sammenhang. Dagegen geben die tieffarbigen Lösungen von Fluorenon und seinen Derivaten in konz. $\mathrm{H}_{2} \mathrm{SO}_{4}$ im Elektronenspinresonanz-Spektrometer (ESR) ein Signal, das auf die Bildung von Radikalen hinweist. Die Farbvertiefung ist aber nicht auf irgendwelche Zersetzungsprodukte der FluorenonDerivate in konz. $\mathrm{H}_{2} \mathrm{SO}_{4}$ zurückzuführen - die Testsubstanz konnte nach Tagen aus den dunklen Lösungen durch Verdünnen mit Wasser in reinster Form (geprüft am UV- und IR-Spektrum) wiedergewonnen werden. Wahrscheinlich entstehen bei der Reaktion mit Säuren Oxoniumsalze, die tief gefärbt sind. Für das Vorliegen von Oxoniumsalzen spricht auch die Tatsache, daß die Carbonylstreckfrequenz $\left(v_{\mathrm{C}-0}\right)$ von I bzw. II in konz. $\mathrm{H}_{2} \mathrm{SO}_{4}$ langwellig verschoben ist. Die Bande bei $18500 \mathrm{~cm}^{-1}$ beim Fluorenon und $20500 \mathrm{~cm}^{-1}$ bei der Fluorenon-1carbonsäure (Abb. 2 und 3) kann so möglicherweise als eine durch Oxoniumsalzbildung entstehende neue „Salzbande“ beschrieben werden, die als solche dann allerdings verhältnismäßig schwach wäre, während die $n \rightarrow \pi^{x}$-Bande kurzwellig verschoben und von intensiveren $\pi \rightarrow \pi^{\mathrm{x}}$-Banden überlagert sein müßte. Form und Intensität der längstwelligen Bande in verschiedenen Säuren sprechen dagegen mehr für eine langwellige Verschiebung der $\mathrm{n} \rightarrow \pi^{\mathrm{x}}$-Bande (vgl. Abb. 2) .

Dieses Ergebnis bei den Fluorenon-Derivaten steht in Widerspruch zur oben zitierten Regel. Wenn aber durch Solvatationseffekte die n-Elektronen stärker gebunden werden als in unpolaren Lösungsmitteln, und gleichzeitig die $\pi$-Elektronen so deformiert werden, daß auch der niederste leere $\pi$-Term sehr stark absinkt, kann die $\mathrm{n} \rightarrow \pi^{\mathrm{x}}$-Bande auch - entgegen der allgemeinen Regel - nach langen Wellen verschoben werden.

Es sei noch erwähnt, daß auch die kristalline Fluorenon-1-carbonsäure ein sehr schwaches ESRSignal gibt, im Gegensatz zum kristallinen Fluorenon und zum Fluorenon-1-carbonsäuremethylester. Wegen der geringen Intensität (ca. $10^{17}$ Elektronen/g) erscheint ein Zusammenhang mit der intensiven Farbe nicht sehr wahrscheinlich.

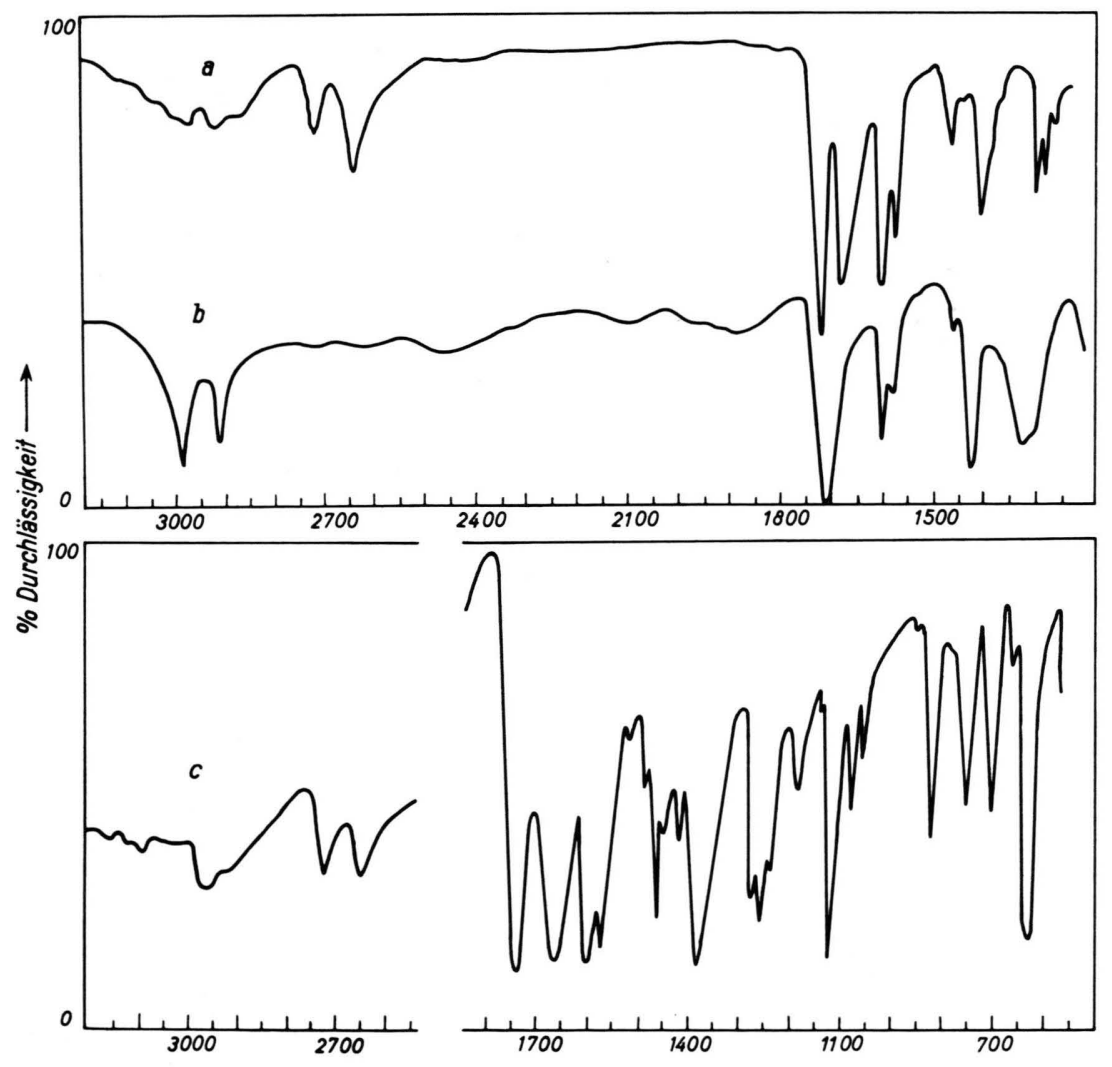

Abb. 5. IR-Spektren der Fluorenon-1-carbonsäure. a) in Chloroform ' (gesättigte Lösung), b) in Dimethylsulfoxyd (gesättigte Lösung), c) in $\mathrm{KBr}$ als Preßling (1 mg Säure pro $300 \mathrm{mg} \mathrm{KBr})$. 


\section{IR-Spektren}

\subsection{Lösungen der Fluorenon-1-carbonsäure}

Die Fluorenon-1-carbonsäure ist in den meisten Lösungsmitteln $\left(\mathrm{CCl}_{4}, \mathrm{CS}_{2}\right.$, Hexan, Äther, Alkohol etc.) nur in so geringen Mengen löslich, daß eine IR-spektroskopische Untersuchung dieser Lösungen kein brauchbares Ergebnis bringt. In Chloroform, in dem die Säure relativ gut löslich ist, gleicht das IR-Spektrum weitgehend dem KBr-Feststoffspektrum der Substanz (vgl. Abb. 5). Es treten charakteristische OH-Schwingungsbanden bei 2670, 2740, 2905 und $2945 \mathrm{~cm}^{-1}$ auf. Die CO-Valenzschwingungen erscheinen bei 1730 und $1690 \mathrm{~cm}^{-1}$, d. h. die Aufspaltung ist geringer als im $\mathrm{KBr}$-Spektrum (1735 und $\left.1665 \mathrm{~cm}^{-1}\right)$. Die IR-Spektren wurden in $\mathrm{CHCl}_{3}$ in NaCl-Küvetten aufgenommen; im Vergleichsstrahl befand sich reines $\mathrm{CHCl}_{3}$. Die für II besonders charakteristischen $\mathrm{OH}-\mathrm{Banden}$ bei 2670 und $2740 \mathrm{~cm}^{-1}$ erscheinen unabhängig von der Konzentration bei der gleichen Wellenzahl und im gleichen Intensitätsverhältnis.

Sehr gut löslich ist die Fluorenon-1-carbonsäure in Dimethylsulfoxyd. Die IR-Spektren lassen sich hier schon in sehr geringer Schichtdicke $(\sim 10 \mu)$ zwischen Irtran-Fenstern aufnehmen. Die scharfen Banden bei 2670, 2740, 2905 und $2945 \mathrm{~cm}^{-1}$ sind verschwunden, dagegen treten bei 2990 und 2910 $\mathrm{cm}^{-1}$ zwei scharfe Banden auf, die aber - wie ein Vergleich mit dem Spektrum des reinen Lösungsmittels zeigt - als symmetrische und asymmetrische CH-Valenzschwingungen der Methylgruppen des Dimethylsulfoxyds zu deuten sind. Außerdem erscheint bei $1900 \mathrm{~cm}^{-1}$ eine breite Bande, desgleichen eine flache Absorptionszone zwischen 2600 und 2400 $\mathrm{cm}^{-1}$ (vgl. Abb. 5), die für II charakteristisch ist.

Die CO-Valenzschwingung ist nicht mehr aufgespalten. Man beobachtet in Dimethylsulfoxyd nur eine intensive Bande bei $1715 \mathrm{~cm}^{-1}$.

\subsection{IR-Untersuchung in KBr-Tabletten}

Wegen der schlechten Löslichkeit der Fluorenon1-carbonsäure und ihrer Derivate in inerten Lösungsmitteln wurden die Substanzen als KBr-Preßlinge vermessen. Das Festsubstanzspektrum von II zeigt im langwelligen Teil des Spektrums mehr und schärfere Banden als das Lösungsspektrum in $\mathrm{CHCl}_{3}$ (Abb. 5). Die für die weiteren Betrachtungen besonders wichtigen $v_{\mathrm{OH}^{-}}$und $v_{\mathrm{CO}}$-Schwingungen sind in beiden Spektren analog.

\subsubsection{Die OH-Schwingungsbanden}

In Abb. 6 ist das OH-Valenzschwingungsgebiet von II und IV wiedergegeben. Die Fluorenon-4-säure (b) zeigt das für dimere Säuren charakteristische Bild - eine breite Bande zwischen 3000 und 2500 $\mathrm{cm}^{-1}$ (von $v_{\mathrm{CH}}$-Schwingungen überlagert) mit dem Maximum bei $3000 \mathrm{~cm}^{-1}$ und kleineren Satellitenbanden nach langen Wellen. Die scharfe Bande geringer Intensität bei $3420 \mathrm{~cm}^{-1}$ könnte von einem kleinen Anteil monomerer Fluorenon-4-säure herrühren. Ganz anders ist das Spektrum der isomeren 1-Säure (a). Auch hier ist zwar der OH-Valenzschwingungsbereich um ca. $800 \mathrm{~cm}^{-1}$ langwellig gegenüber monomeren Säuren verschoben, aber es treten - im Gegensatz zu den dimeren Carbonsäuren - mehrere scharfe Banden auf.

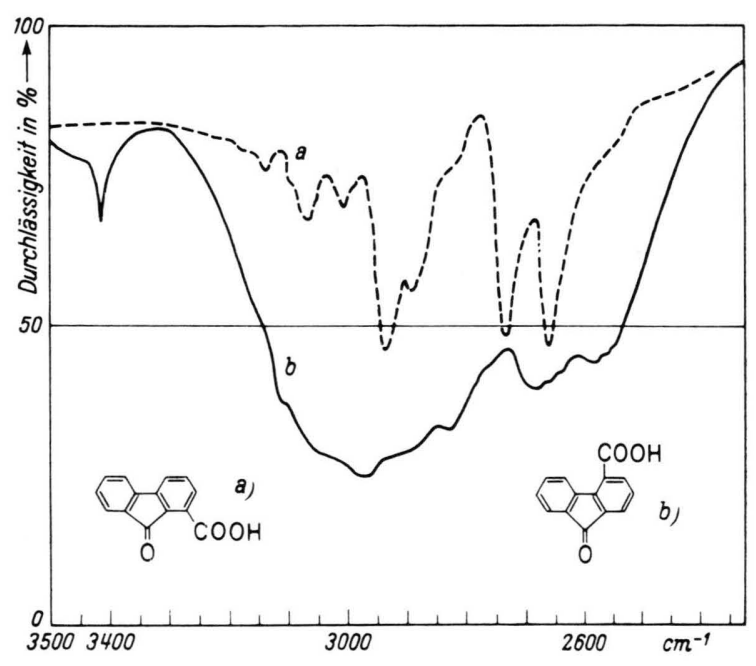

Abb. 6. Vergleich der $v_{\mathrm{OH}^{-}}$und der $v_{\mathrm{CH}^{-}}$-Schwingungen. a) der Fluorenon-1-carbonsäure, b) der Fluorenon-4-carbonsäure.

Um aus der Gesamtheit der Banden zwischen 3000 und $2500 \mathrm{~cm}^{-1}$ die $v_{\mathrm{OH}^{-}}$Banden zu isolieren, wurden die IR-Spektren der Fluorenon-1-carbonsäure und des Silbersalzes der Säure miteinander verglichen. Die Spektren des Silbersalzes und des Methylesters von II können keine OH-Banden aufweisen, die im genannten Schwingungsbereich auftretenden Banden sind als $v_{\mathrm{CH}}$-Schwingungen zuzuordnen (vgl. Abb. 7). Durch Vergleich der Spektren erkennt man, daß die Banden bei 3020 und 3080 $\mathrm{cm}^{-1}$ auf aromatische $v_{\mathrm{CH}}$-Schwingungen zurückzuführen sind. Nur in der freien Säure treten 6 Banden auf: $v=2945,2905,2860,2740,2670$ (und 2580) $\mathrm{cm}^{-1}$; sie gehören zu $v_{\mathrm{OH}^{-}}$Schwingungen. 
Dieser Schluß wird durch Ersatz des Protons der Carboxylgruppe durch Deuterium und Vermessung der Fluorenon-1-deuterocarbonsäure gefestigt (vgl. Abb. 8).

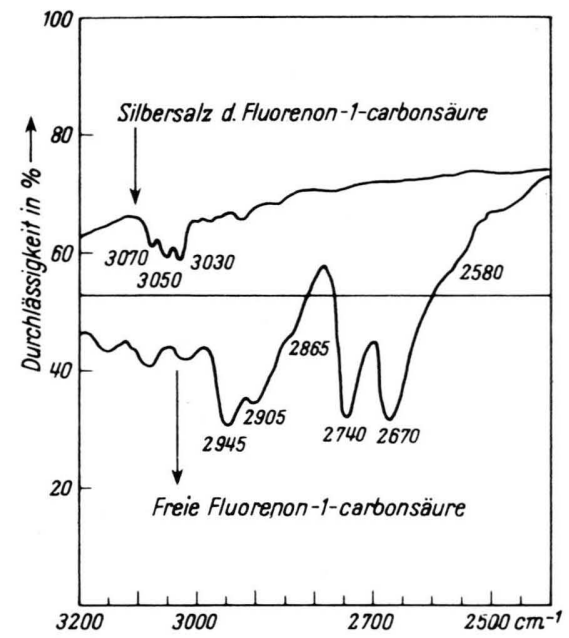

Abb. 7. Isolierung der $v_{\mathrm{OH}}$-Schwingungen in der Fluorenon1-carbonsäure durch Vergleich mit dem Spektrum des Silbersalzes der Fluorenon-1-carbonsäure.

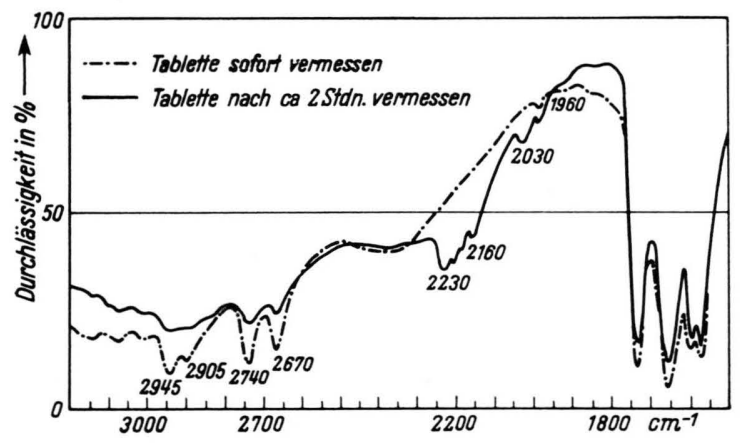

Abb. 8. $v_{0 D}$-Schwingungen der deuterierten Fluorenon-1carbonsäure.

Wird allgemein in einer Hydroxylverbindung das Proton durch Deuterium ersetzt, so ist nach der Beziehung $\bar{v}=(1 / 2 \pi) \cdot \sqrt{f / m}$ mit einer Verschiebung der OH-Frequenz um den Faktor $1 / \sqrt{2}=0,707 \mathrm{zu}$ rechnen. Im IR-Spektrum der frisch deuterierten Carbonsäure beobachtet man neben den Banden bei 2945,2740 und $2670 \mathrm{~cm}^{-1}$, deren Intensität merklich abgeschwächt erscheint, neue Banden bei 2230 , 2150 und $2030 \mathrm{~cm}^{-1}$. Diese Banden sind auf Valenzschwingungen der $\mathrm{O}-\mathrm{D}$-Gruppe zurückzuführen. Auch bei Beachtung aller Vorsichtsmaßregeln ließ sich das Spektrum der vollständig deuterierten Verbindung nicht erhalten. Die neuen Banden ver- schwinden innerhalb von ca. 1 Stde. durch Rückaus tausch des Deuteriums mit einem Wasserstoffatom. Aus der Form der Banden kann man folgern: die Bande bei $2945 \mathrm{~cm}^{-1}$ wird nach 2230, die bei 2900 nach 2160 und die bei 2740 nach $2030 \mathrm{~cm}^{-1}$ durch Deuterierung verschoben. Das gemessene Verhältnis $v_{\mathrm{OH}}: \boldsymbol{v}_{\mathrm{OD}}$ beträgt in diesen Fällen 1,33, was in guter Übereinstimmung mit dem theoretischen, für den harmonischen Oszillator berechneten Wert von 1,41 steht; die geringe Abweichung dürfte von der Anharmonizität herrühren.

Verbindungen, bei denen die gleiche räumliche Orientierung der Carbonylgruppe zur Carboxylgruppe wie in der Fluorenon-1-carbonsäure gegeben ist, weisen, wie die Spektren der 3.4-Benzofluorenon1-carbonsäure und der Fluorenon-1.8-dicarbonsäure zeigen, mehrere intensive $v_{\mathrm{OH}}$-Banden auf. Die 7-Chlorfluorenon-1-carbonsäure ist von gleicher oranger Farbe wie die halogenfreie Säure und hat $v_{\mathrm{OH}}$-Banden bei den gleichen Wellenzahlen. Dagegen zeigt das IR-Spektrum der gelben, in Nachbarstellung der Carboxylgruppe halogensubstituierten 2 Chlorfluorenon-1-carbonsäure zwischen 3000 und $2500 \mathrm{~cm}^{-1}$ keine charakteristischen $v_{\mathrm{OH}}$-Banden, dafür eine verhältnismäßig breite unstrukturierte Bande mit einem Maximum bei $3250 \mathrm{~cm}^{-1}$. Von Tröster ${ }^{2}$ wurde dies als Folge einer starken Wechselwirkung der Hydroxylgruppe mit dem Halogen gedeutet.

\subsubsection{Die Carbonylschwingungsbande}

Die CO-Valenzschwingung in aromatischen Ketonen liegt bei $1660 \mathrm{~cm}^{-1}$. Durch die Ringspannung infolge der Einebnung im Fluorenon wird die Carbonylfrequenz auf $1715 \mathrm{~cm}^{-1}$ erhöht. die $\boldsymbol{v}_{\mathrm{CO}}$ Schwingung der Carbonsäuren liegt bei 1700 bis $1725 \mathrm{~cm}^{-1}$. Durch den Einfluß von Arylsubstituenten wird die Frequenz auf $1675 \mathrm{~cm}^{-1}$ herabgedrückt $\left(v_{\mathrm{CO}}\right.$ der Fluorenon-1-carbonsäure $\left.=1675 \mathrm{~cm}^{-1}\right)$.

Die Fluorenon-4-carbonsäure enthält die beiden CO-Banden an der gleichen Stelle, wie sie einzeln im Spektrum des Fluorenons und der Fluorencarbonsäure (VI) auftreten. In der isomeren Fluorenon1-carbonsäure zeigen sich 2 CO-Banden bei 1665 und $1735 \mathrm{~cm}^{-1}$ (vgl. Abb. 9). Das Spektrum des Silbersalzes der Fluorenon-1-carbonsäure zeigt eine Bande bei $1715 \mathrm{~cm}^{-1}$, die der Carbonylfrequenz im Fluorenon entspricht und außerdem Banden bei 1570 und $1610 \mathrm{~cm}^{-1}$, die asymmetrischen und symmetrischen Streckschwingungen des Carboxylatanions. Die 3.4-Benzofluorenon-1-carbonsäure zeigt 


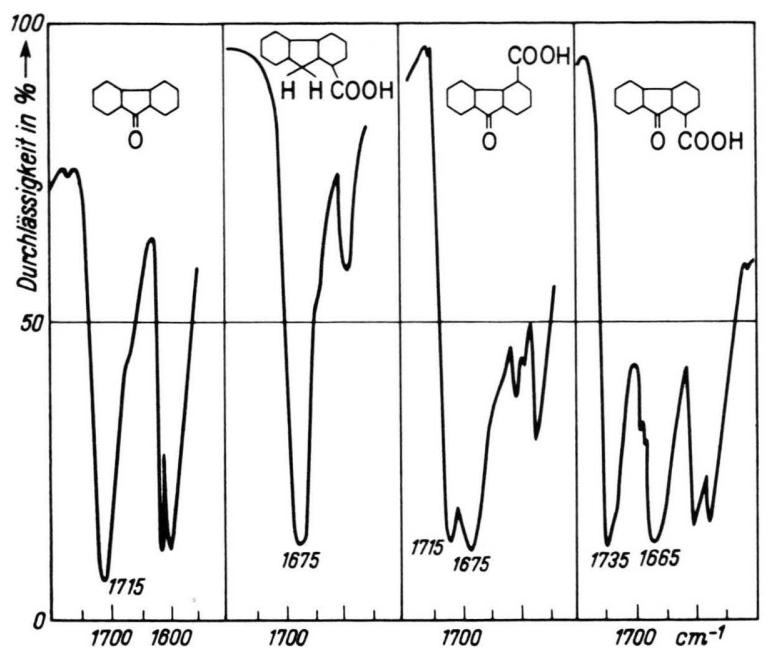

Abb. 9. Zuordnung der $v_{\mathrm{CO}}$-Schwingungen in der Fluorenon1-carbonsäure durch Vergleich mit den $v_{\mathrm{CO}}$-Frequenzen des Fluorenons, der Fluoren-1-carbonsäure und der Fluorenon4-carbonsäure.

ebenfalls zwei CO-Banden bei 1735 und 1665 $\mathrm{cm}^{-1}$. Nach höheren Frequenzen verschoben liegen dagegen die Banden in der 2-Chlorfluorenon-1-carbonsäure $\left(1715\right.$ und $\left.1750 \mathrm{~cm}^{-1}\right)$.

\subsubsection{Abhängigkeit der OH-Banden von der Temperatur}

Aufheizung bzw. Abkühlung der KBr-Tabletten bringt keine Verschiebung in der Lage der OH-Banden (vgl. Abb. 10). Selbst in der Schmelze $\left(200{ }^{\circ} \mathrm{C}\right.$ ) liegen die Banden bei der gleichen Wellenzahl wie bei $-130{ }^{\circ} \mathrm{C}$. Dagegen ist das Intensitätsverhältnis der $\mathrm{OH}-B$ anden untereinander temperaturabhängig (vgl. Abb. 10 und Tab. 1). Die Isolierung der In- tensitäten für die nur schwach ausgeprägten Banden $v_{1}, v_{2}$ und $v_{3}$ ist mit großer Unsicherheit behaftet. Unter „heißen Banden“ versteht man solche, die bei Temperaturerhöhung stärker werden $\left(v_{2}\right.$ und $\left.v_{4}\right)$.

Um IR-Spektren auch bei Temperaturen der flüssigen Luft aufnehmen zu können, wurde ein eigener Küvettenhalter gebaut, der durch ein Kühlmittel auf niedere Temperaturen gebracht werden kann und der durch Wärmeleitung seinerseits den auf ihm angebrachten KBr-Preßling auf die gewünschte Temperatur bringt ${ }^{6}$.

\subsubsection{Oberschwingungen der Fluorenon-1-carbon- säure}

Im nahen IR wurden die Fluorenon-Derivate mit den Spektralphotometern Zeiss PMQ II und Beckman DU untersucht. Man erhielt keine scharfen Banden, die als Oberschwingungen der $v_{\mathrm{CO}^{-}}$bzw. $v_{\mathrm{OH}}$ Frequenzen hätten gedeutet werden können. Einige scharfe Absorptionslinien im nahen IR konnten mittels Vergleichsspektren als Oberschwingungen der aromatischen $v_{\mathrm{CH}}$ zugeordnet werden.

\section{Diskussion der IR-Spektren}

\subsection{D i e $\mathrm{OH}-\mathrm{B}$ anden}

Bei der Fluorenon-1-carbonsäure kann man erwarten, daß sie, wie praktisch alle Carbonsäuren, als cyclisches Dimeres vorliegt, also intermolekulare Wasserstoffbrücken bildet. Innerhalb der Molekel kann es jedoch, im Gegensatz zur isomeren 4-Carbonsäure, auch zur Ausbildung eines inneren, sterisch besonders begünstigten 7-Ringes kommen.

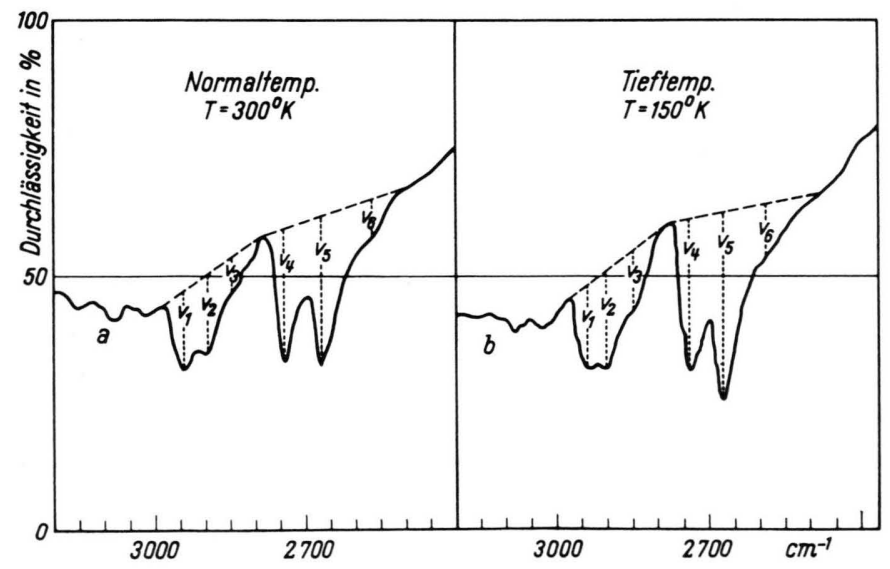

Abb. 10. Intensitäten der $v_{0 H}$-Banden. a) bei Zimmertemperatur, b) bei $-120^{\circ} \mathrm{C}$. II in $\mathrm{KBr}$.

${ }^{6}$ G. Demmering, Diss., TH München 1962. 
Während nun das Spektrum der 4-Säure die für Carbonsäuredimere charakteristischen breiten, intensiven $\mathrm{OH}$-Absorptionsbanden zeigt, weist die Fluorenon-1-carbonsäure zwischen 3000 und $2500 \mathrm{~cm}^{-1}$ OH-Banden auf, die für Carbonsäuren ungewöhnlich sind. Die langwellige Verschiebung um $800 \mathrm{~cm}^{-1}$ gegenüber der monomeren OH-Bande (ca. 3400 $\mathrm{cm}^{-1}$ ) weist auf das Vorliegen starker Wasserstoffbrücken hin. Diese langwelligen $\boldsymbol{v}_{\mathrm{OH}}$-Banden, deren Gesamtintensität deutlich geringer als für Dimere mit intermolekularen H-Brücken ist, sind gut ausgeprägt und verhältnismäßig scharf strukturiert (vgl. Abb. 6) .

Dies und das Auftreten einer scharfen $\gamma_{\mathrm{OH}}$-Bande bei $840 \mathrm{~cm}^{-1}$, im Gegensatz zur Fluorenon-4-carbonsäure, spricht für das Vorliegen sehr starker intramolekularer H-Brücken in der roten Fluorenonl-carbonsäure.

Wie das Spektrum in $\mathrm{CHCl}_{3}$ zeigt, liegen diese intramolekularen Brücken auch in den gelben Lösungen vor. Die $v_{\mathrm{OH}}$-Banden sind, wie Versuche in $\mathrm{CHCl}_{3}$ zeigen, konzentrationsunabhängig, eine Tatsache, die mit dem Vorliegen intermolekularer Brükken nicht vereinbar wäre. Zum gleichen Schluß kommt man aus der Temperaturunabhängigkeit der $\mathrm{OH}$-Schwingungsfrequenzen der Fluorenon-1-carbonsäure.

Aus dem Fehlen einer monomeren $v_{\mathrm{OH}}$-Bande bei $3400 \mathrm{~cm}^{-1}$, dem Auftreten einer sehr schwachen Absorptionszone zwischen 2600 und $2400 \mathrm{~cm}^{-1}$ sowie einer intensiven Bande bei $1950 \mathrm{~cm}^{-1} \mathrm{kann}$ man folgern, daß im Dimethylsulfoxyd (Abb.5) die intramolekulare Brücke der Fluorenon-1-carbonsäure gebrochen wurde und die Säure dafür intermolekulare H-Brücken mit den polaren Lösungsmittelmolekülen eingeht; der Sauerstoff des Dimethylsulfoxyds muß also stärker basisch, d. h. ein stärkerer Protonenakzeptor als der Carbonylsauerstoff sein. Die Bande bei $1950 \mathrm{~cm}^{-1}$ ist als eine besonders langwellige $v_{\mathrm{OH}}$-Schwingung anzusehen.

\section{$5.2 \mathrm{D}$ i e $v_{\mathrm{CO}}-\mathrm{B}$ ande}

Wie die $\mathrm{OH}$-, so werden auch die $v_{\mathrm{CO}}$-Frequenzen durch Wasserstoffbrückenbindung beeinflußt. Fuson und $\operatorname{Josien}^{7}$ ordnen ohne nähere Begründung die längerwellige Bande bei $1665 \mathrm{~cm}^{-1}$ der Carboxylgruppe und die bei $1735 \mathrm{~cm}^{-1}$ der Ketogruppe zu. Wir ordnen die $v_{\mathrm{CO}}$-Frequenzen umgekehrt zu. Wie aus Abb. 9 hervorgeht, finden sich in der Fluorenon4-carbonsäure zwei CO-Banden an den gleichen Stellen wie für die Keto- $v_{\mathrm{CO}}$-Bande im Fluorenon (1715 $\mathrm{cm}^{-1}$ ) und die Carboxyl- $\boldsymbol{v}_{\mathrm{CO}}$-Bande in der Fluorenon-1-carbonsäure $\left(1675 \mathrm{~cm}^{-1}\right)$. In der Fluorenon4-carbonsäure beeinflussen sich beide CO-Frequenzen nicht; anders in der Fluorenon-1-carbonsäure, ein weiterer Hinweis für das Vorliegen intramolekularer H-Brücken in letzterer.

Es ist einzusehen, daß durch H-Brückenbindung die Ketofrequenz von ursprünglich $1715 \mathrm{~cm}^{-1}$ auf $1665 \mathrm{~cm}^{-1}$ langwellig verschoben werden kann. Um aber auch eine Erklärung dafür zu finden, daß die $v_{\mathrm{CO}}$-Frequenz der Carboxylgruppen von 1675 auf $1735 \mathrm{~cm}^{-1}$ erhöht wird, muß man bedenken, daß Carbonsäuren allgemein als Dimere vorliegen, die $v_{\mathrm{CO}}$-Frequenz also schon durch intermolekulare $\mathrm{H}$ Brücken geweitet ist, d. h. die $v_{\mathrm{CO}}$-Bande der ,monomeren" Fluorenoncarbonsäure liegt tatsächlich bei kürzeren Wellen. Die Erhöhung der $v_{\mathrm{CO}}$-Carboxylfrequenz läßt sich mit der Annahme einer intramolekularen H-Brücke erklären.

5.3 Beeinflussung der intramolekularen H-Brücken durch elektronenanziehende Substituenten

$$
\text { in } 2 \text {-Stellung }
$$

Während die kristalline 7-Chlorfluorenon-1-carbonsäure die gleiche orangerote Farbe wie die halogenfreie Säure hat, ist die 2-Chlorfluorenon-1-carbonsäure gelb, desgleichen die 2-Nitroverbindung. Aus der geringen langwelligen Verschiebung der $v_{\mathrm{OH}}$-Bande in der 2-Chlorfluorenon-1-carbonsäure (bei $3200 \mathrm{~cm}^{-1}$ ) ergibt sich, daß durch den elektronegativen Substituenten in 2-Stellung die Ausbildung einer starken intramolekularen H-Brücke verhindert wird. Da Banden zwischen 3000 und 2500 $\mathrm{cm}^{-1}$ fehlen, sind auch keine intermolekularen Brükken vorhanden. Dieser Schluß wird durch die Lage der CO-Frequenzen gestützt. Die Bande bei 1710 $\mathrm{cm}^{-1}$ entspricht der nur schwach langwellig verschobenen Ketofrequenz; durch die elektronenanziehende Wirkung des Halogens kommt es zu einer Verringerung des CO-Abstandes in der Carboxylgruppe und damit zu einer Erhöhung der $v_{\mathrm{CO}}$-Frequenz von 1735 in der Flurenon-1-carbonsäure auf $1750 \mathrm{~cm}^{-1}$ in der 2-Chlorfluorenon-1-carbonsäure.

7 N. Fuson u. M. L. Josien, J. Amer. chem. Soc. 73, 478 [1957]; Bull. Soc. chim. France 19, 389 [1952]. 


\section{Versuchsweise Deutung der OH-Banden durch ein Doppelpotential}

Zimmermann $^{8}$ deutete die relativ große Zahl von $\nu_{\mathrm{NH}}$-Banden beim festen Imidazol durch die Annahme eines Potentials mit zwei ungefähr gleich tiefen Minima für die Protonen der Wasserstoffbrücke. Seine Vorstellungen scheinen auch zur Deutung der hier beschriebenen Spektren geeignet.

Bei der Fluorenon-1-carbonsäure (II) kann man nach Zimmermann ${ }^{8}$, Dodd und Stephenson ${ }^{9}$ die intramolekulare Wasserstoffbrückenbindung durch die beiden tautomeren Grenzstrukturen a) und b) beschreiben.

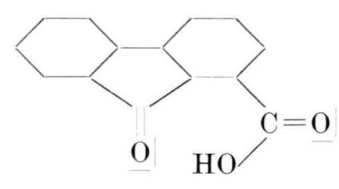

a)

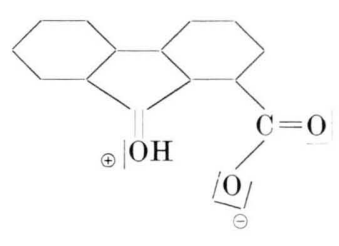

b)
Das Proton bewegt sich innerhalb der Wasserstoffbrückenbindung in einem Potential mit zwei Minima, wobei das eine Minimum in der Nähe des Protonendonators, das zweite Minimum in der Nähe des Elektronendonators liegt. Ein häufiger Wechsel des Protons zwischen zwei Potentialminima läßt sich spektroskopisch an einer Termaufspaltung der Schwingungszustände nachweisen.

Es soll untersucht werden, ob sich die $6 v_{\mathrm{OH}}$-Banden von II in ein Termschema einordnen lassen, das dem von $Z_{\text {Immermans }}{ }^{8}$ für Imidazol aufgestellten Schema entspricht. Nach den Vorstellungen von Batuev ${ }^{10}$ lassen isch alle diese OH-Banden auf Kombinationsschwingungen der relativ energiereichen $\mathrm{OH}-$ Valenzschwingungen $(\mathrm{H}$ gegen $\mathrm{O})$ mit den energieärmeren Schwingungen der Wasserstoffbrükkenbindung ( $\mathrm{OH}$ gegen $\mathrm{O})$ erklären. Wenn es gelänge, von der Fluorenon-1-carbonsäure im Kristall Ramanfrequenzen, nahe der Erregerlinie, zu bestimmen, wäre damit die Möglichkeit gegeben, die Frequenzen der Wasserstoffbrücken-Schwingung, die, je nach Stärke der Brücke, bei etwa $50 \mathrm{~cm}^{-1}$ liegen sollen, zu ermitteln. Diese niederen Ramanfrequenzen

\footnotetext{
8 H. Zimmermann, Z. Elektrochem., Ber. Bunsenges. physik.
} Chem. 65, 821 [1961]. müßten dann als Differenzen der $v_{\mathrm{OH}}$-Banden auftreten. Die Bestimmung derartig niederer Ramanfrequenzen an farbigen Kristallen kann jedoch zur Zeit noch nicht durchgeführt werden; damit ist die Möglichkeit genommen, wie beim Imidazol den Abstand der unteren Terme direkt festzustellen.

Es wurde deshalb versucht, diesen Abstand aus der Temperaturabhängigkeit des Intensitätsverhältnisses zweier Banden durch Anwendung des B o l t z m a n n - Faktors zu ermitteln.

Für das Intensitätsverhältnis zweier Banden a und $b$, die zu Anregungen von verschiedenen Termen aus in den gleichen Endterm gehören, gilt bei den Temperaturen $T_{1}$ bzw. $T_{2}$ :

$$
\left(\frac{I_{\mathrm{a}}}{I_{\mathrm{b}}}\right)_{T_{1}}:\left(\frac{I_{\mathrm{a}}}{I_{\mathrm{b}}}\right)_{T_{2}}=e^{-\frac{\Delta E}{k}\left(\frac{1}{T_{1}}-\frac{1}{T_{2}}\right)}
$$

wobei: $\Delta E=$ Abstand der Ausgangsterme, gesucht, $k=\mathrm{B}$ o l t z m a n n - Konstante, $I_{\mathrm{a}}=$ Intensität der Bande a, $I_{\mathrm{b}}=$ Intensität der Bande b.

Nach dieser Methode wurden die Energiedifferenzen $\Delta E$ der $v_{\mathrm{OH}}$-Banden aus Tab. 1 bestimmt. Die bei $2735 \mathrm{~cm}^{-1}$ und $2945 \mathrm{~cm}^{-1}$ liegenden Banden, die mit $v_{1}$ und $v_{4}$ bezeichnet werden, sind ,heiße Banden“. Übergänge, die solchen „heißen Banden“ entsprechen, gehen von höheren Termen aus, die durch Temperatursteigerung stärker besetzt werden können.

Die nach der Methode über den BoltzmannFaktor ermittelten Energiedifferenzen $\Delta E$ liegen in der Größenordnung, wie wir sie für die energiearmen Schwingungen der Wasserstoffbrückenbindung $(\mathrm{OH} \ldots \mathrm{O})$ in der Fluorenon-1-carbonsäure erwarten und wie sie beim Imidazol als Ramanfrequenzen gemessen werden konnten ${ }^{8}$. Diese Analogie ist ein Hinweis dafür, daß man auch die $v_{\mathrm{OH}^{-}}$ Banden der Fluorenon-1-carbonsäure in ein Termschema einordnen kann und daß die von ZimmerMANN am Beispiel des Imidazols abgeleiteten Vorstellungen über die Theorie der Wasserstoffbrückenbindung allgemeiner angewendet werden können. Zur Aufstellung eines vollständigen Termschemas wären jedoch Ramanmessungen unbedingt erforderlich.

9 R. E. Dodd u. G. W. Stephenson, in: D. Hadzi, Hydrogen Bonding. Pergamon-Press, S. 339, London 1959.

10 M. I. Batuev, Zhurn. Fiz. Khim. 23, 1399 [1949]. 


\section{Vorstellungen zur Erklärung der Farb- vertiefung der Fluorenon-1-carbonsäure}

Aus den IR-spektroskopischen Untersuchungen der Fluorenoncarbonsäuren und ihrer Derivate ergibt sich u. a., daß nur diejenigen Verbindungen eine Farbanomalie zeigen, bei denen eine intramolekulare Wasserstoffbrückenbindung besteht. Ein Vergleich der UV- und IR-Spektren zeigt andererseits, daß das Vorliegen von intramolekularen Wasserstoffbrücken - wie z. B. bei der Fluorenon-1-carbonsäure in unpolaren Lösungsmitteln - nicht unbedingt Farbvertiefung zur Folge hat.

Es besteht also kein direkter Zusammenhang zwischen Wasserstoffbrückenbindung und Farbvertiefung.

Die Auswertung der UV- und IR-Spektren des Fluorenons und der Fluorenon-1-carbonsäure ergab, daß in aciden Lösungsmitteln die Farbvertiefung von gelb bzw. orange nach rot-violett auf die Ausbildung eines Oxoniumsalzes zurückzuführen ist. Es ist daher zu vermuten, daß auch im Kristall die Farbvertiefung der Fluorenon-1-carbonsäure gegenüber ihrer Lösung in unpolaren Lösungsmitteln durch das Vorliegen von Oxoniumstrukturen bedingt wird. Derartige innere Oxoniumsalze - wie sie bereits von $\mathrm{H}_{\text {ANTZSCH }}{ }^{1}$ erwähnt werden - können sich dann ausbilden, wenn die Carbonyl- und Carboxylgruppe, wie in der Fluorenon-1-carbonsäure, benachbart sind. Im Kristall „springt“ dann das Proton dér Carboxylgruppe zum Sauerstoff der Ketogruppe unter Ausbildung einer polaren Struktur b), siehe oben.

Mit dieser Deutung der Farbvertiefung steht die Tatsache in Übereinstimmung, daß die $v_{\mathrm{C} 0}$-Bande der Ketogruppe von ursprünglich $1715 \mathrm{~cm}^{-1} \mathrm{im}$ Fluorenon sowohl in der kristallinen Fluorenon-1carbonsäure als auch in konz. $\mathrm{H}_{2} \mathrm{SO}_{4}$ auf etwa 1660 $\mathrm{cm}^{-1}$ um $55 \mathrm{~cm}^{-1}$ langwellig verschoben erscheint, während sie in der gelben Chloroformlösung nur um $25 \mathrm{~cm}^{-1}$ verschoben, bei $1690 \mathrm{~cm}^{-1}$, auftritt.

In der energiereicheren Schmelze und in Lösungsmitteln dürften dann keine inneren Oxoniumsalze, wohl aber, wie erwähnt, Wasserstoffbrücken vorliegen.

Daraus soll nun nicht gefolgert werden, daß für den kristallinen Zustand die Wasserstoffbrückenbindung ausschließlich durch die polare Form (inneres Oxoniumsalz), für die Lösung durch die unpolare Form beschrieben wird, wohl aber, daß der Anteil der polaren Grenzstruktur (oder das Gewicht der polaren Grenzstruktur) an der Wasserstoffbrückenbindung im roten Kristall wesentlich größer ist als in den gelben Lösungen.

\section{Experimentelles}

\subsection{A p paratur}

Die UV-Absorptionsspektren wurden mit einem Zeiss-Spektral-Photometer PMQ II, die IR-Spektren mit einem IR-Spektrometer Perkin-Elmer 221 aufgenommen.

\section{2 $\mathrm{Substanzen}$}

\section{Deuterierung der Fluorenon-1-carbonsäure}

Um in der Fluorenon-1-carbonsäure den aciden Wasserstoff durch Deuterium zu ersetzen, wurde vom gelben Silbersalz der Säure ausgegangen, aus dem durch Zusatz von DCl die zinnoberrote deuterierte freie Säure und ein flockiger Niederschlag von $\mathrm{AgCl}$ entsteht. Fluorenon-1-carbonsäure tauscht das auf diese Weise eingefügte Deuterium sehr schnell wieder gegen Protonen aus. Es ist daher erforderlich, die Verbindung möglichst rasch zu trocknen, mit $\mathrm{D}_{2} \mathrm{O}$ zu waschen und mit $\mathrm{KBr}$ zu Tabletten zu verarbeiten, das vorher mit $\mathrm{D}_{2} \mathrm{O}$ befeuchtet und rasch getrocknet wurde.

\begin{tabular}{|c|c|c|c|c|c|c|}
\hline $\begin{array}{c}\text { Tempe- } \\
\text { ratur } \\
{\left[{ }^{\circ} \mathrm{K}\right]}\end{array}$ & $\begin{array}{c}v_{1} \\
2945\end{array}$ & $\begin{array}{c}\nu_{2} \\
2905\end{array}$ & $\begin{array}{c}\nu_{3} \\
2860\end{array}$ & $\begin{array}{c}\nu_{4} \\
2740\end{array}$ & $\begin{array}{c}\nu_{5} \\
2670\end{array}$ & $\begin{array}{c}v_{6} \\
2580 \mathrm{~cm}^{-1}\end{array}$ \\
\hline $\begin{array}{l}300 \\
150\end{array}$ & $\begin{array}{c}54 \\
45 \\
, \text { heiß }\end{array}$ & $\begin{array}{l}53 \\
52\end{array}$ & $\begin{array}{l}29 \\
33\end{array}$ & $\begin{array}{c}90 \\
82,5 \\
\text {,heiß“، }\end{array}$ & $\begin{array}{l}100 \\
100\end{array}$ & $\begin{array}{c}29 \\
28,5\end{array}$ \\
\hline
\end{tabular}

Tab. 1. Intensitäten der $v_{\mathrm{OH}}$-Banden der Fluorenon-1-carbonsäure bei verschiedenen Temperaturen, bezogen auf $\mathrm{I}\left(v_{5}\right)=100$.

\begin{tabular}{|c|cc|}
\hline $\begin{array}{c}\text { Kombinierte } \\
\text { Banden }\end{array}$ & $\begin{array}{c}\text { Termdifferenzen } \\
\Delta E \text { berechnet } \\
{\left[\mathrm{cm}^{-1}\right]}\end{array}$ \\
\hline$v_{1} / v_{2}$ & $43 \quad(\approx 40)$ \\
$v_{1} / \nu_{4}$ & $22 \quad(\approx 20)$ \\
$v_{1} / v_{5}$ & $38 \quad(\approx 40)$ \\
$v_{2} / \nu_{4}$ & $21,5(\approx 20)$ \\
$v_{2} / \nu_{5}$ & 1 & $(\approx 0)$ \\
$v_{4} / v_{5}$ & $22 \quad(\approx 20)$ \\
\hline
\end{tabular}

Tab. 2. Termdifferenzen $\Delta E$, berechnet aus der Temperaturabhängigkeit der Bandenintensitäten nach dem B o l t z m a n n - Faktor.

Die Verfasser danken dem Direktor des PhysikalischChemischen Instituts, Herrn Professor Dr. G. Scheibe, für die Förderung dieser Arbeit, Herrn Professor A. Sieglitz für wertvolle Hinweise und Überlassung einiger Substanzen, der De u t s chen For schung gemeinschaft und dem Fonds der Chemi$\mathrm{s}$ chen Industrie für materielle Unterstützung. 